PALABRAS CLAVE

Crecimiento económico

Macroeconomía

Exportaciones

Inversiones

Tipos de cambio

Devaluación

Modelos econométricos

Indicadores económicos

México

Carlos Ibarra

Profesor Titular

Departamento de Economía

Universidad de las Américas, Puebla

œ carlos.ibarra@udlap.mx
REVISTA DE LA CEPAL 95 AGOSTO 2008

\section{La paradoja del crecimiento lento de México}

\author{
Carlos Ibarra
}

$\mathrm{E}$

ste trabajo analiza el problema de crecimiento económico lento de México. Descompone el aumento del producto por el lado de la demanda e ilustra la incidencia fundamental de la atonía de la inversión. Sostiene, con respaldo econométrico, que esa atonía se explica en parte por la apreciación real del peso durante la desinflación y su efecto adverso en la rentabilidad de la inversión. Por último, muestra que el problema se ha complicado por una merma a largo plazo de la razón $\mathrm{PIB} /$ capital. 


\section{I}

\section{Introducción}

Tras la "década perdida" — los años 1980—, las perspectivas económicas de México mejoraron en forma espectacular, conforme el país recibía un gran volumen de capital extranjero y se transformaba en un importante exportador de manufacturas. La evolución positiva se registró tras las reformas estructurales por las que se liberalizó el régimen de comercio y se privatizó la mayoría de los sectores de actividad económica. Se renegoció la deuda externa del país, se consolidaron las finanzas públicas y, con excepción de un revés transitorio en 1995, se observó un claro y exitoso empeño por reducir la inflación. Pero, a pesar del dinamismo de las exportaciones, de la sustancial entrada de capital extranjero y de la estabilización de la economía, México ha encarado un problema persistente de crecimiento lento.

Varios analistas han ofrecido posibles explicaciones de esta aparente paradoja. En un trabajo señero, Moreno-Brid (1999) sostiene que el crecimiento de México enfrenta una restricción externa más rigurosa debido a la mayor elasticidad de las importaciones respecto del producto interno bruto (PIB) después de la liberalización del comercio. ${ }^{1}$ Según Blecker (2007), la restricción se vio a veces reforzada por variaciones en las corrientes de capital y el ciclo económico de los Estados Unidos, y por la recurrente apreciación del peso.
Otros autores señalan los efectos adversos de la prolongada restricción del crédito en el país, derivada del avance insuficiente en las reformas del poder judicial y de la banca. ${ }^{2}$

Este trabajo busca ofrecer una interpretación complementaria del problema de crecimiento lento de México. El punto de partida es la comprobación de que desde fines de los años 1980, la gestión macroeconómica mexicana se ha centrado en la reducción de la inflación. El prolongado "modo desinflacionario" de esa gestión, y en particular de la política monetaria, tiende a apreciar el peso en términos reales y, por ende, a reducir la rentabilidad de la inversión. El efecto negativo de la apreciación del peso en la rentabilidad se combina con una caída a largo plazo de la relación producto/capital. Como resultado, la inversión agregada no refleja el dinamismo de las exportaciones ni permite alcanzar altas tasas de crecimiento económico.

$\mathrm{El}$ análisis se desarrolla de la manera siguiente: en la sección II se describe el desempeño macroeconómico reciente de México; en la sección III se estima el aporte de las exportaciones y la inversión al crecimiento del PIB; en la sección IV se examina principalmente la relación entre ganancias, inversión y tipo de cambio real, y en la sección V se presentan las conclusiones.

\section{II \\ El desempeño macroeconómico reciente de México}

Después del estallido de la crisis de la deuda en 1982, el crecimiento económico de México se detuvo. En el período 1982-1988 el PIB mexicano subió apenas un 0,2\% por año. Sin embargo, el sector exportador se amplió

\footnotetext{
$\square$ El autor agradece los valiosos comentarios de Julio López, Jaime Ros, un evaluador anónimo y a los participantes de la Novena Conferencia Poskeynesiana Internacional y a la VII Conferencia de las Américas, así como el apoyo financiero del Consejo Nacional de Ciencia y Tecnología (CONACYT, proyecto 47140-S).

${ }^{1}$ Véase también López y Cruz (2000) y Pacheco-López (2005).
}

con rapidez, estimulado por la fragilidad del mercado interno y la liberalización del comercio implantada en 1986-1987. En comparación con las cifras de 1960-1977, la participación de las exportaciones totales en el PIB se duplicó con creces. Particularmente impresionante fue el aumento de las exportaciones de manufacturas, a una tasa media anual de $18,2 \%$ por año (cuadro 1 ).

\footnotetext{
2 Véase Bergoeing y otros (2001) y Tornell, Westermann y Martínez (2004).
} 


\begin{tabular}{|c|c|c|c|c|c|}
\hline & $1960-1977$ & $1978-1981$ & $1982-1988$ & 1989-1994 & $1996-2007$ \\
\hline \multicolumn{6}{|l|}{ Exportaciones totales $(\%)^{\mathrm{a}}$} \\
\hline Tasa de crecimiento & $6,69^{\mathrm{aa}}$ & 18,76 & 8,26 & 7,82 & 8,60 \\
\hline Participación en el PIB & 5,31 & 7,43 & 12,14 & 14,86 & 34,35 \\
\hline \multicolumn{6}{|l|}{ Exportaciones de manufacturas (\%) } \\
\hline Tasa de crecimiento ${ }^{\mathrm{b}}$ & $11,26^{\mathrm{bb}}$ & 9,23 & 18,20 & 12,14 & 8,42 \\
\hline $\begin{array}{l}\text { Entradas de capital extranjero } \\
\quad(\text { Participación en el } \mathrm{PIB}, \%)\end{array}$ & 1,30 & 5,12 & 2,36 & $6,28^{\mathrm{dd}}$ & 3,43 \\
\hline Tasa de crecimiento del PIB $(\%)^{\mathrm{e}}$ & 6,23 & 9,11 & 0,22 & 3,91 & 3,58 \\
\hline \multicolumn{6}{|l|}{ Inflación del IPC $(\%)^{\mathrm{f}}$} \\
\hline Tasa máxima & $29,1(1977)$ & $27,9(1981)$ & $131,8(1987)$ & 26,7 (1990) & $34,4(1996)$ \\
\hline Variación anual media, en puntos porcentuales & $3,4^{\mathrm{ff}}$ & $-0,3$ & 12,3 & $-17,9$ & $-2,6$ \\
\hline \multicolumn{6}{|l|}{ Costo relativo de la mano de obra $(1990=100)^{\mathrm{g}}$} \\
\hline Promedio & $\ldots$ & $\ldots$ & $\ldots$ & 116,8 & 115,4 \\
\hline Mínimo & $\ldots$ & $\ldots$ & $\ldots$ & 92,2 (1989) & 75,4 (1996) \\
\hline Máximo & $\ldots$ & $\ldots$ & $\ldots$ & $138,8(1993)$ & $135,7(2007)$ \\
\hline Variación media anual (\%) & $\cdots$ & $\cdots$ & $\cdots$ & 8,0 & 4,8 \\
\hline \multicolumn{6}{|c|}{ Tipo de cambio real multilateral basado en el IPC $(1990=100)^{\mathrm{h}}$} \\
\hline Promedio & $68,7^{\mathrm{gg}}$ & 76,1 & 100,3 & 87,8 & 76,3 \\
\hline Mínimo & $63,5(1975)$ & $62,0(1981)$ & $78,3(1985)$ & $100,6(1989)$ & $102,9(1996)$ \\
\hline Máximo & 87,7 (1977) & $87,1(1978)$ & $134,4(1987)$ & $73,4(1993)$ & $61,17(2002)$ \\
\hline Variación media anual (\%) & $4,0^{\text {hh }}$ & $-8,1$ & 11,2 & $-6,0$ & $-3,2$ \\
\hline
\end{tabular}

Fuente: Banco Mundial (2005) e INEGI.

a Tasas medias anuales según datos de las cuentas nacionales a precios locales constantes.

b Tasas medias anuales según datos de balanza de pagos deflactados por el índice de precios al productor de los Estados Unidos. Fuente: Banco Mundial (2005) y Banco de México (1982-2007).

c Tasas medias anuales según datos de balanza de pagos en dólares corrientes. Fuente: Banco Mundial (2005) y Banco de México (19822007).

d Préstamos bancarios, inversión extranjera directa e inversión de cartera. Promedio de corrientes trimestrales en dólares de los Estados Unidos. El PIB fue convertido a dólares usando el tipo de cambio nominal. Fuente: Banco Mundial (2005), INEGI y Banco de México.

e Tasas medias anuales. Fuente: INEGI.

f Fuente: Banco de México.

g Razón del costo unitario de la mano de obra México/Estados Unidos, sector de manufacturas. Fuente: INEGI.

h Fuente: Banco de México. Una disminución del índice del tipo de cambio real indica una apreciación.

aa 1961-1977.

bb $1963-1977$.

cc 1962-1977.

dd No incluye el primer trimestre de 1994.

ee 1970-1977.

ff 1971-1977.

gg 1968-1977.

hh $1969-1977$.

A fines de la década de 1980, una combinación de factores - entre ellos un nuevo plan de estabilización, la renegociación definitiva de la deuda externa del país y el renovado acceso de los países en desarrollo al mercado mundial de capital— cambió las perspectivas de la economía mexicana. Aumentó notablemente la inversión extranjera, de 2,4\% del PIB en 1982-1988 a 6,3\% en 1989-1994. El sector exportador mantuvo su dinamismo, con un crecimiento medio anual de las exportaciones de 7,8\%. La exportación de manufacturas, en particular, se incrementó a un promedio anual de $12,1 \%$ y llegó a constituir el $75 \%$ de las exportaciones de bienes. Sin 
embargo, a pesar del auge de las exportaciones y de la gran afluencia de capital, el crecimiento se desaceleró luego de haber alcanzado un máximo de 5\% en 1990. Así, la tasa media de crecimiento del PIB en 1989-1994 fue moderada: $3,9 \%$.

Como es bien sabido, México sufrió una crisis cambiaria en diciembre de 1994, seguida de una crisis financiera de grandes proporciones en 1995. Hubo ingentes salidas de capital, debidas sobre todo a la reversión de anteriores inversiones de cartera. El PIB acabó por bajar más de 6\% en 1995. Pero la actividad económica se recuperó con rapidez y en 1996 el PIB creció más de $5 \%$. Las entradas de capital se reanudaron y llegaron a un promedio anual de 3,4\% del PIB en 1996-2007. ${ }^{3}$

El sector de exportación de manufacturas siguió expandiéndose, aunque a un ritmo menor que a comienzos del decenio de 1990. En 1996-2007 las manufacturas llegaron a representar el $85 \%$ de la exportación de bienes y en 1998 México ya era el cuarto exportador mundial de manufacturas (Lall, 2000). Junto con crecer, el sector exportador exhibió avances cualitativos. La canasta de exportaciones llegó a tener una composición similar a la que se observa en países con un ingreso per cápita superior al de México (Hausmann, Hwang y Rodrik, 2007, gráfico 4). Las exportaciones del país corresponden en su mayor parte a bienes de nivel tecnológico medio $\mathrm{o}$ alto (Lall, 2000), y se han diversificado desde que entró en vigor el Tratado de Libre Comercio (TLC) de América del Norte (Feenstra y Kee, 2007).

Pese a la reanudación de la inversión extranjera y al desarrollo cuantitativo y cualitativo de las exportaciones, el crecimiento medio anual del PIB en 1996-2007 fue de solo 3,6\%. El problema no es que México no haya podido alcanzar tasas relativamente elevadas de crecimiento del PIB — de hecho las tuvo en 1990, 1997 y 2000—, sino que esas tasas han durado muy poco. Hay factores que reducen la tasa de crecimiento y que tienden a mantenerla baja. En este trabajo se argumenta que uno de esos factores es la evolución del tipo de cambio real, por su efecto en la rentabilidad y la inversión.

En tanto que la economía no pudo mantener tasas elevadas de crecimiento del PIB, la gestión macroeconómica y en particular la política monetaria apuntaron

\footnotetext{
${ }^{3}$ Aunque esta cifra es de casi la mitad de las entradas de capital registradas a comienzos de la década de 1990, sigue siendo elevada desde la perspectiva histórica del país; por ejemplo, en el período de elevado crecimiento de 1960-1977, los ingresos de capital equivalían a $1,3 \%$ del PIB.
}

a reducir la inflación (Ramos y Torres, 2005; Galindo y Ros, 2008; Ibarra, 2008a). La economía enfrentó un serio problema inflacionario casi toda la década de 1980, con una inflación que subía en promedio unos 12,3 puntos porcentuales por año, llegando a $131,8 \%$ en 1987. La inflación media anual del período 19821988 fue de $88 \%$.

La desinflación empezó en 1988. Al principio fue rápida, ya que la tasa anual de inflación bajó de alrededor de $180 \%$ a comienzos de 1988 a menos de $20 \%$ un año más tarde. Después el proceso se desaceleró: la tasa media anual de inflación en el período 1989-1994 fue de 16,9\%, con una disminución a 6,9\% en 1994.

El plan desinflacionario se derrumbó en diciembre de 1994, ante la necesidad de abandonar una banda cambiaria explícita para dejar flotar el peso. Tras la posterior depreciación de la moneda repuntó la inflación. Pero el fenómeno duró poco y casi inmediatamente la economía entró en una nueva etapa desinflacionaria. La inflación alcanzó un máximo de 34,4\% en 1996 y un mínimo de 3,6\% en 2006; en promedio, se redujo 2,6 puntos porcentuales por año.

En suma, la gestión macroeconómica de México ha estado "en modo desinflacionario" desde fines de los años 1980, lo que ha repercutido con fuerza en el tipo de cambio. Dado el sesgo restrictivo de la política monetaria, la tasa de depreciación monetaria tiende a ir a la zaga de la diferencia de inflación entre México y los Estados Unidos. Como lo ilustra el índice real efectivo calculado por el Banco de México (cuadro 1, últimas líneas), el resultado es que el peso tiende a apreciarse en términos reales.

La apreciación del peso contribuyó al éxito de la desinflación (Ibarra, 2003), pero tuvo un efecto negativo en la rentabilidad del sector de bienes transables. La evolución del costo unitario de la mano de obra en las manufacturas (medido en dólares y en relación con los Estados Unidos) ilustra este efecto. En 1988, inmediatamente después de un importante episodio de liberalización del comercio, el índice del costo de la mano de obra era de $82,9(1990=100)$, pero en el primer período desinflacionario (1989-1994) aumentó sostenidamente a una tasa de $8 \%$ por año, alcanzando un máximo de 138,8\% en 1993. La fuerte depreciación monetaria nominal de 1995 lo redujo al nivel observado a mediados de la década de $1980(1996=75,4)$, pero al reanudarse la desinflación el costo relativo de la mano de obra creció nuevamente, registrando un nivel de 135,7 en el 2007 (cuadro 1). 


\section{III}

\section{Las exportaciones, la inversión y el crecimiento del producto interno bruto en México}

¿Cuál es el origen del magro crecimiento de la economía mexicana? Según se reconoce ampliamente en las publicaciones sobre crecimiento, puede ser útil abordar el tema a diferentes niveles. Para ello se suele distinguir entre los determinantes próximos y los determinantes más profundos del crecimiento del PIB. Las descomposiciones del crecimiento son un ejemplo del primero de estos enfoques. En los párrafos siguientes se analiza la evolución del PIB agregado según la contribución de los diferentes componentes de la demanda agregada. ${ }^{4}$

La descomposición del PIB es útil para analizar la experiencia de crecimiento de México porque pone de relieve elementos tales como la sostenida expansión de las exportaciones registrada desde fines de los años 1980, la tendencia al alza en la tasa de importación después de la liberalización del comercio y el auge del consumo de comienzos de la década de 1990. Cabe imaginar que estos factores son importantes desde una perspectiva de mediano plazo. Está, por ejemplo, la cuestión de determinar cuál fue el efecto neto de la exportación en el crecimiento agregado, en un sentido meramente contable, habida cuenta del casi simultáneo aumento de la tasa de importación. A medida que las exportaciones aumentaban, las importaciones desplazaban a la producción local.

El punto de partida es la simple identidad contable que iguala el PIB total $Y$ con la demanda agregada, que es la suma del consumo privado y del gobierno $(C+G)$, la inversión $I$ y la exportación neta $(X-M)$. La identidad puede reordenarse así:

$$
Y=d_{0}(I+X), d_{0}=\frac{1}{s+m}
$$

donde $d_{0}$, el multiplicador keynesiano de la demanda, es por definición igual a la inversa de la suma de la tasa de ahorro interno $[s=(Y-C-G) / Y]$ y la tasa de importación $(m=M / Y)$.

El crecimiento del PIB tiene que ser respaldado por una expansión de la inversión y las exportaciones,

\footnotetext{
${ }^{4}$ Véase en Berg y Taylor (2000) una metodología similar y en Ros y Lustig (2000) su aplicación a México.
}

aunque también pueden incidir variaciones sostenidas del multiplicador. El gráfico 1 muestra que, después de una relativa estabilidad desde al menos la década de 1960, el multiplicador de la demanda inició un descenso impresionante en 1987, pasando de 3,6 en 1986 a 1,6 en el 2007.

Por definición, las variaciones del multiplicador reflejan la influencia conjunta de la tasa de ahorro y la tasa de importación. La tasa de ahorro ha fluctuado en torno a 0,20. La sostenida baja del multiplicador de la demanda se explica por la evolución de la tasa de importación, que pasó de 0,084 en 1986 a 0,46 en el 2007. Después de los principales episodios de liberalización del comercio en 1986-1987 y en 1994, hubo pronunciados aumentos de la tasa de importación. En el gráfico 2 se muestra que todos los componentes de la tasa compartieron esta tendencia. ${ }^{5}$

\section{GRÁFICO 1}

\section{México: multiplicador de la demanda y sus componentes, $1960-2007$}

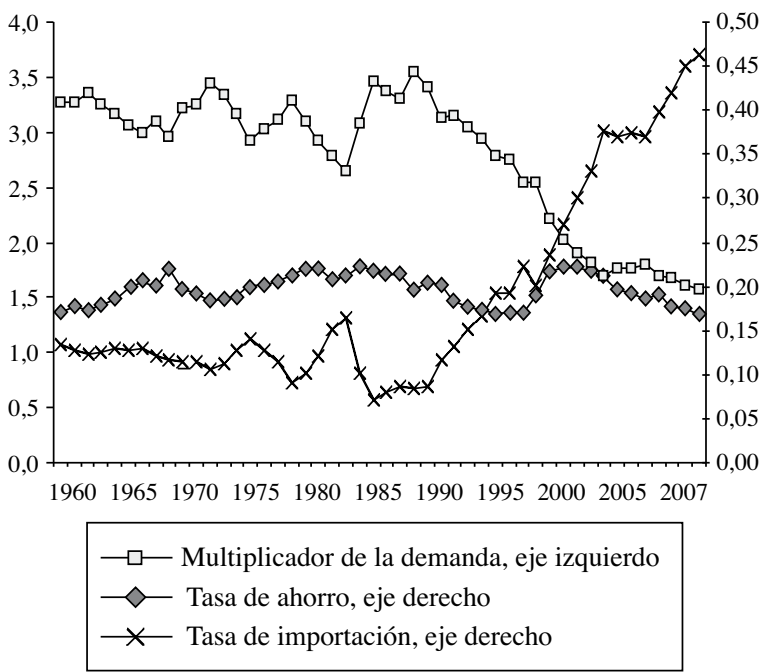

Fuente: cálculos del autor con datos de cuentas nacionales del Banco Mundial (2005) y del INEGI.

\footnotetext{
${ }^{5}$ Véase en el Apéndice una definición de estos componentes.
} 


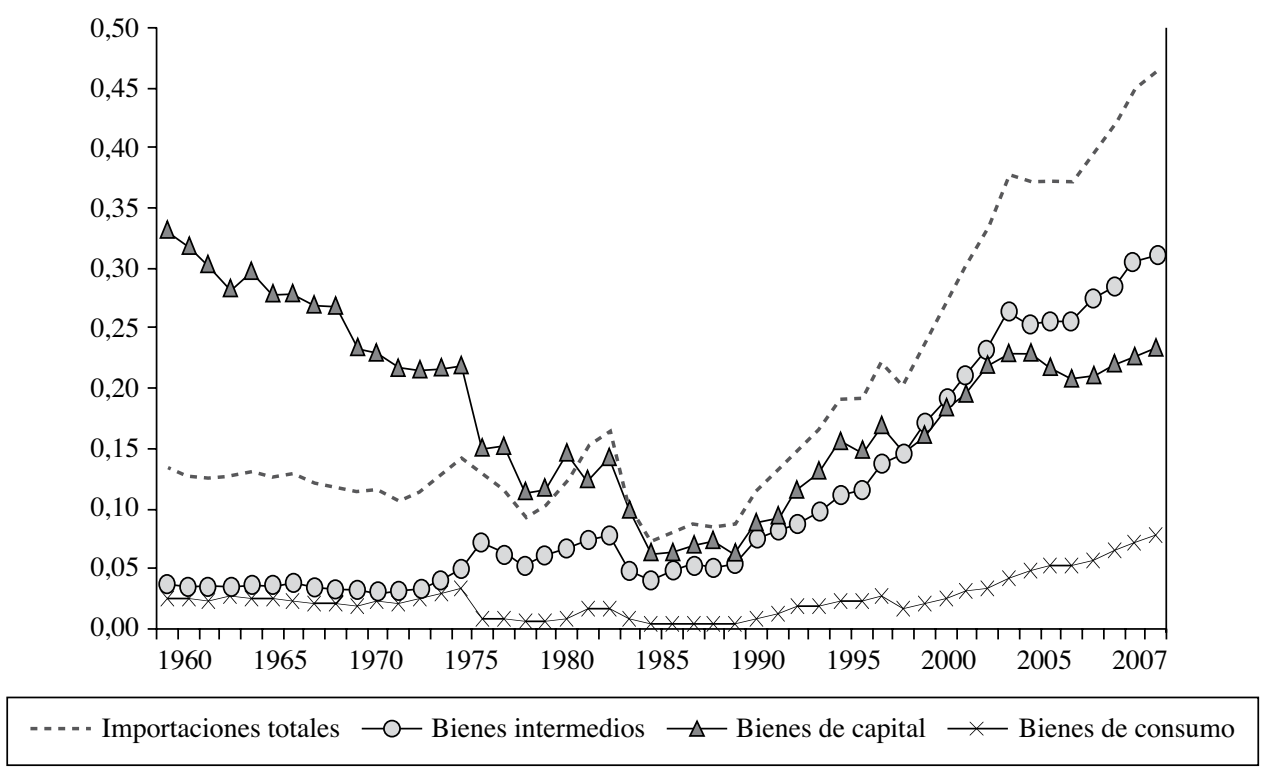

Fuente: cálculos del autor basados en datos del Banco de México (comercio exterior) y Banco Mundial (2005) y del INEGI (cuentas nacionales).

Aparte de la liberalización del comercio, las variaciones del tipo de cambio real son, sin duda, una influencia posible en la tasa de importación. El gráfico 3 presenta la evolución conjunta de la tasa de importación y del índice del tipo de cambio real basado en el índice de precios al consumidor (IPC); con el fin de lograr una mayor eficacia visual, el gráfico presenta el índice invertido, para que un aumento indique una apreciación real de la moneda. Existe una asociación positiva sustancial entre las dos series, con un coeficiente de correlación de 0,5024 . La correlación del tipo de cambio real con tipos específicos de importaciones oscila entre 0,4318 para los bienes intermedios y 0,6222 para los bienes de capital (en el caso de estos últimos, desde 1978, dejando de lado la tendencia a la baja registrada por la tasa de importación en el contexto de una industrialización sustitutiva de las importaciones).

Habría que someter a una prueba rigurosa el vínculo entre el tipo de cambio real y la tasa de importación. Pero la evidencia anterior sugiere que la tendencia de la moneda a apreciarse en términos reales podría haber acentuado el efecto de la liberalización del comercio, de manera que podría haberse producido un aumento "excesivo" de la tasa de importación (Moreno-Brid, Santamaría y Rivas, 2005a y 2005b).

El gráfico 4 exhibe los aportes, ajustados por el multiplicador, de la inversión y la exportación desde 1960. El crecimiento del PIB en la parte inicial de la muestra estaba respaldado por el crecimiento de la inversión. Aplicando la ecuación (1), se puede calcular que alrededor del $80 \%$ del crecimiento acumulativo del PIB en 1960-1977 puede atribuirse a la inversión.

La contribución de los distintos componentes de la demanda agregada varió en el período posterior a la liberalización y las exportaciones pasaron a ser la principal fuente de crecimiento del PIB. Entre 1988 y 2007, este experimentó un aumento de 829.100 millones de pesos de 1993; el $93 \%$ correspondió a exportación y solo $7 \%$ a inversión (también en este caso, previo ajuste de ambas variables a las fluctuaciones del multiplicador). A primera vista, el hecho de que México no haya registrado un fuerte crecimiento económico desde fines de la década de 1980 puede atribuirse a la falta de dinamismo de la inversión. 6,7

\footnotetext{
${ }^{6}$ Más detalladamente: la tasa media de crecimiento de la inversión real en este período fue de 5\%, y estuvo prácticamente contrarrestada por una disminución acumulativa del multiplicador de la demanda de 50\%. La tasa media de crecimiento de la inversión ajustada por el multiplicador fue de $1 \%$; la cifra correspondiente para las exportaciones fue de 5,6\%. Las conclusiones sobre la incidencia de los componentes de la demanda agregada en el crecimiento del PIB dependen de la duración del período que se considere. Después de la primera ronda de liberalización del comercio, el crecimiento del PIB estaba determinado por la inversión, con un aporte mínimo de las exportaciones (Ros, Draisma y otros, 1996; Ros y Lustig, 2000). La situación se revirtió después de que se aprobara el Tratado de Libre Comercio (TLC) de América del Norte.

${ }^{7}$ En una conclusión afín, Blyde y Fernández-Arias (2004) calculan que la relación capital-trabajo de México en los años 1990 era más
} 
GRÁFICO 3

México: tipo de cambio real y tasa de importación, 1960-2005

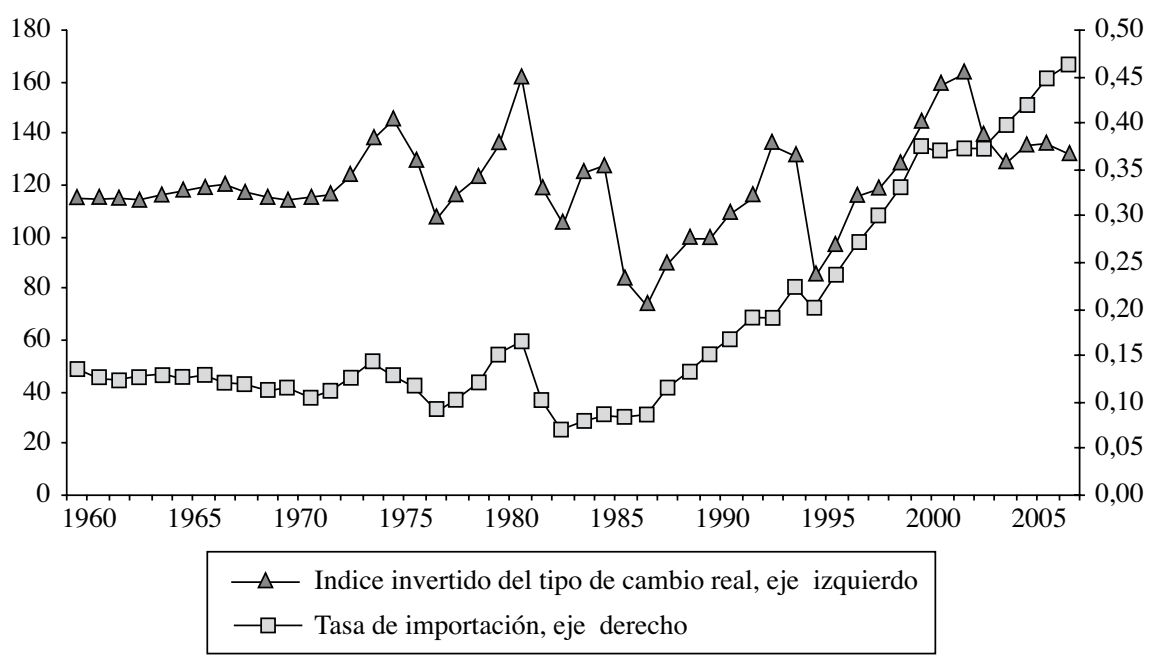

Fuente: cálculos del autor con datos del Fondo Monetario Internacional (International Financial Statistics) y Banco de México (tipo de cambio real), y del Banco Mundial (2005) e INEGI (cuentas nacionales).

GRÁFICO 4

México: descomposición del producto interno bruto, 1960-2007

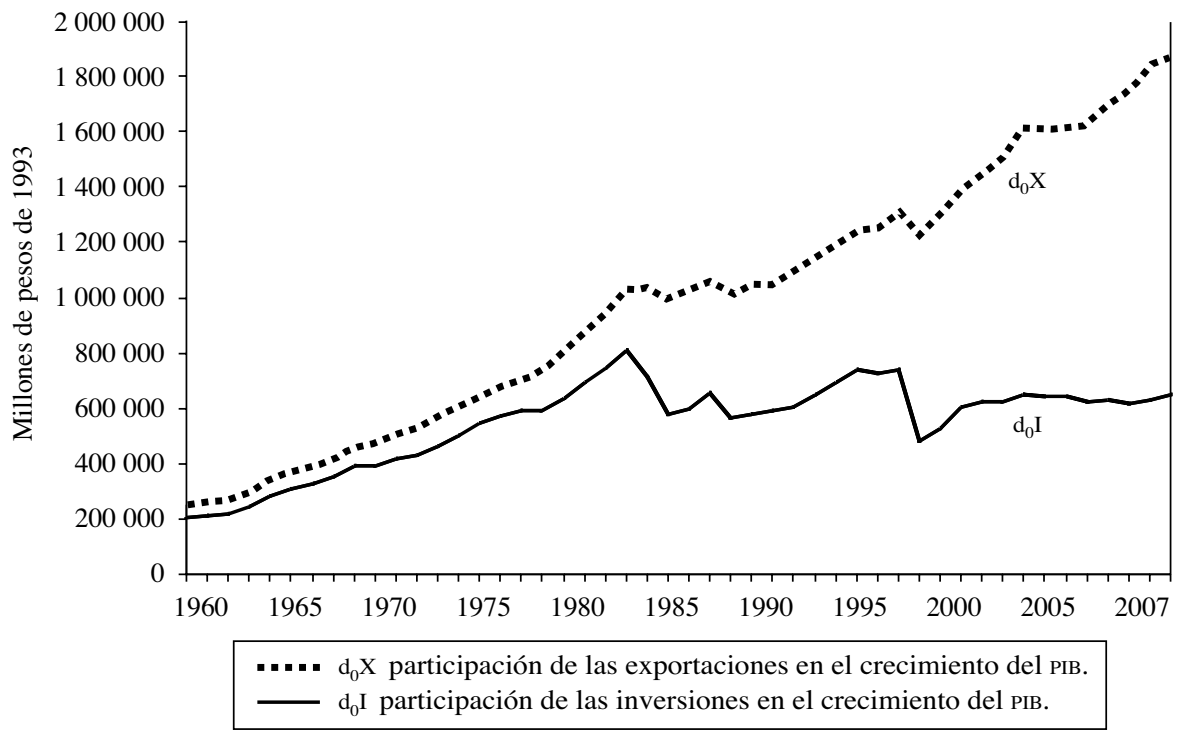

Fuente: cálculos del autor con datos de cuentas nacionales del Banco Mundial (2005) y del INEGI.

Desde el punto de vista de la demanda agregada, el ritmo relativo de expansión de la inversión y las exportaciones es irrelevante; lo que importa es el volumen global de la

de $20 \%$ inferior al nivel que cabía prever por el estado de desarrollo del país; en América Latina, solo Guatemala estaba en una posición más desventajosa. demanda. Sin embargo, del lado de la oferta, la distinción es importante. A diferencia de la exportación, la inversión crea capacidad instalada directamente. De manera que la falta de dinamismo de la inversión también limita indirectamente el crecimiento del PIB, al crear estrangulamientos que pueden desacelerar el aumento de la exportación y estimular aún más la tasa de importación. 
La descomposición anterior se basa en la ecuación (1), que no distingue entre gasto privado y gasto público. En un análisis más detallado, es posible separar el gasto del gobierno y considerar la influencia de las variaciones en la tasa impositiva. Empezando de nuevo a partir de la identidad del PIB, se puede escribir:

$$
Y=d_{1}\left(I^{P}+I^{G}+G+X\right), d_{1}=\frac{1}{s^{p}+t+m},
$$

donde $I^{P}$ e $I^{G}$ representan la inversión privada y la inversión pública; ahora el multiplicador de la demanda depende de la tasa de ahorro privado $s^{P}=(Y-T-C) / Y$, en la que $T$ son los impuestos; de la tasa impositiva $(t=T / Y)$ y de la tasa de importación.

El gráfico 5 ilustra la evolución del multiplicador de la demanda redefinido y de sus componentes desde 1980. También en este caso, el multiplicador sigue una tendencia a la baja desde el segundo quinquenio de los años 1980, atribuible al aumento de la tasa de importación. La tasa de ahorro privado y la tasa impositiva tienen un marcado comportamiento cíclico. ${ }^{8}$ La tasa de ahorro privado registra variaciones ligeramente mayores que la tasa de ahorro total indicada más atrás en el gráfico 1; ello refleja el hecho de que la evolución de la tasa de impuestos tiende a reproducir inversamente la del ahorro privado (calculada sin incluir la tasa de impuestos).

El gráfico 6 muestra la contribución al PIB de los distintos componentes de la demanda agregada desde 1980. Lo nuevo que sugiere el gráfico es que en México la falta de dinamismo de la inversión privada, que ha contribuido a moderar el crecimiento del PIB, se ha complicado por el estancamiento de la inversión pública.

Varios autores han planteado la posibilidad de que el sector externo, por la vía del aumento de la tasa de importación, esté imponiendo una restricción al crecimiento de México. Pero, por otra parte, México ha recibido corrientes de capital extranjero bastante significativas desde los primeros años de la década de 1990, lo que tiende a atenuar esa posible restricción. Así, el comportamiento de la demanda de inversión resulta revelador si se lo examina desde el punto de vista de la balanza de pagos.

De acuerdo con la identidad de la balanza de pagos, en un período dado las corrientes de capital extranjero que recibe un país deben ser iguales a la suma de las salidas de capital interno, la acumulación de reservas de divisas por parte del banco central y el déficit en cuenta corriente:

Entradas de capital extranjero = salidas de capital interno

+ acumulación de reservas + déficit en cuenta corriente,

GRÁFICO 5

México: multiplicador de la demanda y sus componentes, 1980-2007

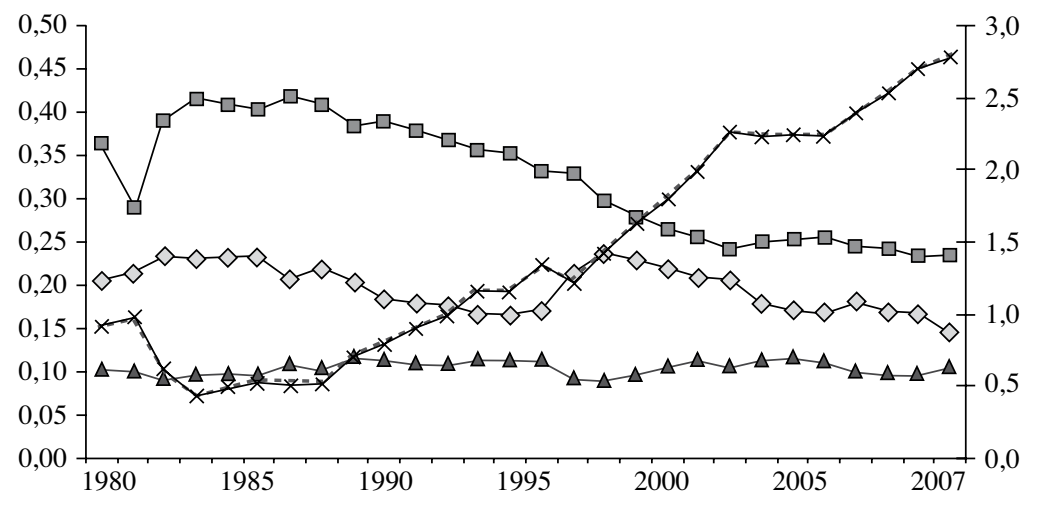

$\checkmark \checkmark$ Tasa de ahorro privado $\longrightarrow$ Tasa de impuestos
$\longrightarrow \longleftarrow$ Tasa de importación $\longrightarrow-$ Multiplicador de la demanda, eje derecho

Fuente: cálculos del autor con datos del Banco de México (finanzas públicas) y del Banco Mundial (2005) y el INEGi (cuentas nacionales).

${ }^{8}$ La tasa impositiva incluye todo el ingreso del sector público por la
actividad económica no petrolera clasificado como impuesto (bási- camente, el impuesto sobre la renta y sobre el valor agregado) más los impuestos a la gasolina. 
GRÁFICO 6

México: descomposición del producto interno bruto, 1980-2007

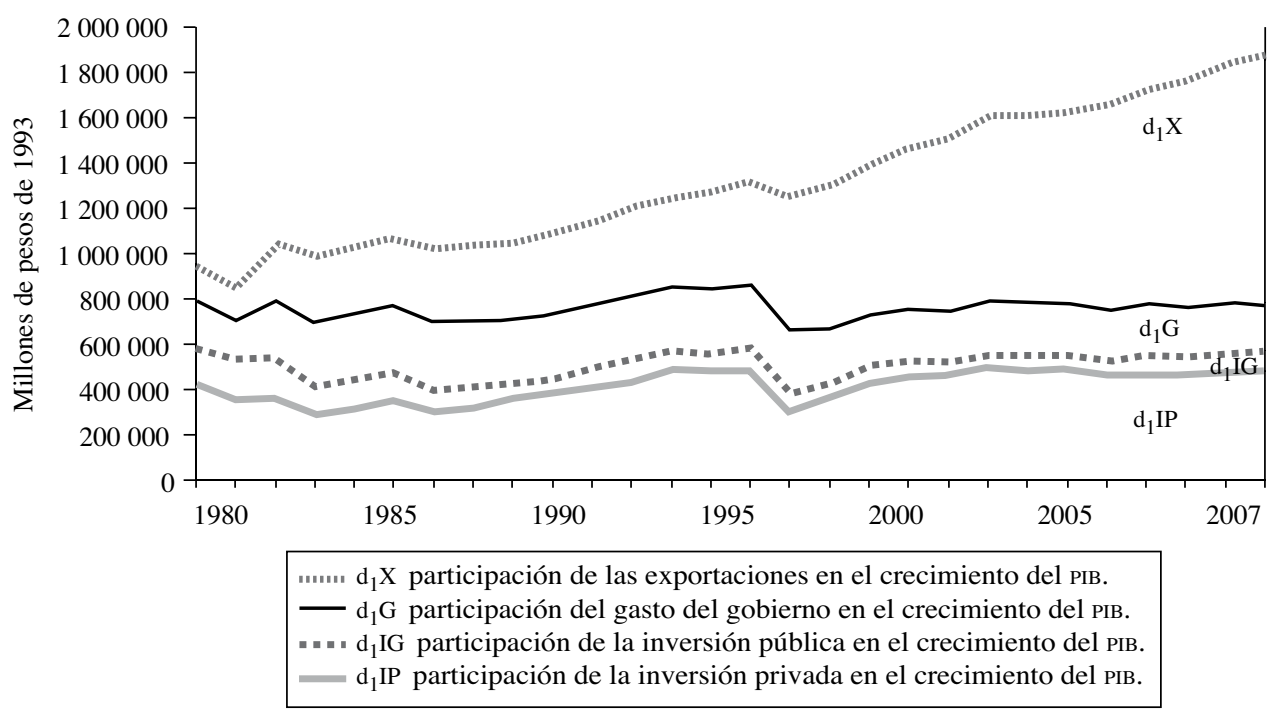

Fuente: cálculos del autor con datos del Banco de México (finanzas públicas) y del Banco Mundial (2005) y el INEGI (cuentas nacionales).

en tanto que el déficit en cuenta corriente debe ser igual a la diferencia entre la inversión y el ahorro internos.

El gráfico 7 ofrece información sobre algunos períodos (primer trimestre de 1983 a cuarto trimestre de 1989, primer trimestre de 1990 a tercer trimestre de 1994 y primer trimestre de 1996 a cuarto trimestre del 2007). La situación inicial del sector externo es bien conocida. Las entradas de capital extranjero eran relativamente reducidas ( $1,7 \%$ del PIB en promedio) y la economía se veía obligada a transferir recursos al exterior en forma de un superávit en cuenta corriente $(0,7 \%$ del PIB). Desde una perspectiva de balanza de pagos, estos recursos se gastaron en una moderada acumulación de reservas $(0,6 \%$ del PIB, en promedio) y fundamentalmente en salida de capital al exterior ( $1,8 \%$ del PIB). Durante este período, la tasa de inversión (fija) fue de $18,5 \%$ del PIB.

El contexto macroeconómico cambió en forma espectacular en los años siguientes. Una combinación de factores locales y externos causó una gran afluencia de capital extranjero, el que alcanzó un promedio de $7,3 \%$ del PIB. Hubo un cambio importante del saldo en cuenta corriente, que dio lugar a un déficit de 5,3\% del PIB. Pese a la gran afluencia de capital, la tasa media de inversión en el período 1990-1994 fue de solo 19,2\% del PIB, es decir, menos de un punto por encima de la tasa del período anterior. El gran cambio del saldo en cuenta corriente tuvo como principal contrapartida una reducción de la tasa de ahorro interno.

En 1996-2007, las entradas de capital extranjero se redujeron en promedio a 3,4\% del PIB, pero seguían estando muy por encima de las observadas en la década de 1980. Casi un cuarto de la afluencia de capital se acumuló en calidad de reservas. El déficit en cuenta corriente registró un promedio de solo $1,8 \%$ del PIB, muy por debajo del 5,3\% de principios de los años 1990. Como contrapartida del menor déficit corriente, hubo una recuperación de la tasa de ahorro interno; por su parte, la tasa de inversión varió ligeramente, pasando de $19,2 \%$ a $19,9 \%$ del PIB. ${ }^{9}$

En resumen, durante el período 1996-2007 la tasa media de inversión estuvo menos de 1,5\% del PIB por encima de la de los "años perdidos" de 1983-1989. Este ajuste moderado se produjo en medio de sustanciales entradas de capital extranjero.

\footnotetext{
${ }^{9}$ La gran acumulación de reservas de este período sugiere que, aunque la posible restricción externa al crecimiento haya aumentado por el incremento de la tasa de importación, esa restricción no ha operado.
} 


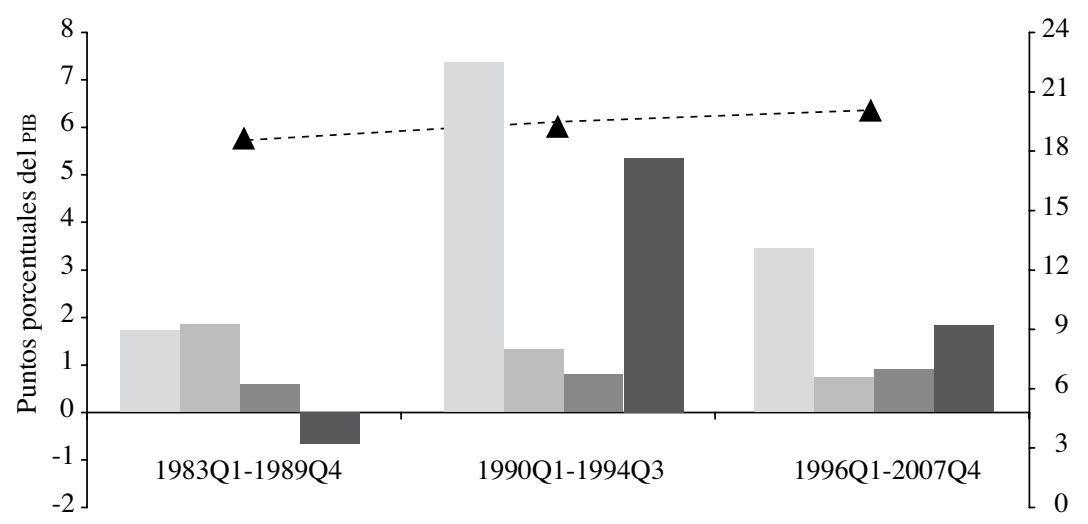

$\square$ Entradas de capital extranjero $\square$ Salidas de capital al exterior $\square$ Acumulación de reservas

Déficit en cuenta corriente --- - Tasa de inversión (eje derecho, como \%)

Fuente: cálculos del autor con datos del Banco de México, del Banco Mundial (2005) y del INEGI (producto interno bruto en pesos corrientes, cambiado a dólares utilizando el tipo de cambio nominal), y del Fondo Monetario Internacional (International Financial Statistics) y del INEGI (tasa de inversión).

\section{IV}

\section{La inversión y la tasa de ganancia}

El débil crecimiento de la economía mexicana puede atribuirse, en un primer enfoque, a la falta de dinamismo de la inversión en un contexto caracterizado por la desinflación y la tendencia del peso a apreciarse en términos reales. En esta sección se examina formalmente el efecto del tipo de cambio real en la rentabilidad del sector manufacturero y, por ese conducto, en la inversión. ${ }^{10}$

Para justificar el análisis empírico, supongamos que los precios fijados por empresas que operan en competencia imperfecta dependen de un margen de ganancia (tal vez variable) y del costo unitario de la mano de obra, según la fórmula:

$$
\frac{P}{S}=(1+u) \Omega, \Omega=a \frac{W}{S}
$$

donde $P$ es el índice de precios internos en moneda local, $u$ el margen de ganancia, $a$ la mano de obra empleada por unidad de producto, $W$ el salario nominal y $\Omega$ el costo unitario de la mano de obra. La ecuación se divide por el tipo de cambio nominal $S$ (definido como pesos por dólar) para convertir a dólares el precio interno y el costo unitario de la mano de obra.

Es posible formular una ecuación similar a la (4) para la economía extranjera, indicando con un asterisco las variables externas. Dividiendo la ecuación del precio interno por su contraparte extranjera, y reordenando, obtenemos:

$$
\frac{\Omega}{\Omega^{*}}=\left(\frac{1+u^{*}}{1+u}\right)\left(\frac{P}{S P^{*}}\right)
$$

\footnotetext{
${ }^{10}$ Usando datos correspondientes al período que va del primer trimestre de 1981 al segundo trimestre del 2000, Lederman, Menéndez y otros (2003) muestran que en México un aumento de la variabilidad del tipo de cambio real tiende a deprimir la tasa de inversión. Mucho se ha debatido el efecto del tipo de cambio real en el volumen y la tasa de crecimiento
}

del PIB. Algunos autores han detectado efectos predominantemente contradictorios. Al respecto véase Kamin y Rogers (2000) y las referencias contenidas en ese trabajo; véase asimismo el punto de vista opuesto en Galindo y Ros (2008), Ibarra (2008b) y Blecker (2007). 
La ecuación (5) indica que un incremento del costo unitario relativo de la mano de obra del país debe ir acompañado de una reducción de su margen de ganancia relativo o de un aumento de sus precios relativos. La reducción del margen de ganancia produciría una merma de la participación de las ganancias en el PIB (z) según la fórmula:

$$
z=\frac{u}{1+u}
$$

El gráfico 8 ilustra la evolución en México del costo relativo de la mano de obra y los precios al consumidor relativos (resumidos más atrás en el cuadro 1). Recordemos que la primera serie corresponde a la relación del costo unitario de la mano de obra manufacturera en dólares entre México y los Estados Unidos y que la segunda serie corresponde al índice del tipo de cambio real multilateral del Banco de México basado en el IPC. Con miras a realzar la eficacia visual del gráfico, se ha invertido el índice original de modo que el aumento indique una apreciación real.

Existe una sólida asociación entre las dos series, como lo sugiere la ecuación (5), con un coeficiente de correlación de 0,8311 durante el período 1985-2007.
Las variaciones de los costos relativos y de los precios relativos están estrechamente conectadas. Un tipo de cambio desalineado - es decir, que no aísla los costos relativos frente a las variaciones relativas en los salarios nominales y las productividades - podría causar una desaceleración de las exportaciones y una pérdida de participación en el mercado.

Podemos examinar el efecto, calculando una regresión del crecimiento de las exportaciones sobre el tipo de cambio real, teniendo en cuenta el crecimiento del mercado. ${ }^{11}$ El cuadro 2 presenta los resultados de la prueba de raíz unitaria sobre las variables utilizadas en el análisis econométrico. La hipótesis de raíz unitaria es rechazada para casi todas las variables, en particular por la prueba de Phillips-Perron.

Algunas variables tienen valores límite en la prueba ampliada de Dickey-Fuller. La participación de las ganancias en el valor agregado de las manufacturas probablemente no sea estacionaria o tal vez lo sea en torno a una tendencia lineal. Debido a esta ambigüedad, las regresiones incluyen los resultados de las pruebas de raíz unitaria aplicadas a los residuos de la versión de largo plazo de las ecuaciones estimadas. Como se sabe, se puede descartar la posibilidad de una regresión espuria cuando la hipótesis de raíz unitaria para los residuos es rechazada.

Las regresiones se calcularon con series trimestrales correspondientes al período 1988-2007, es decir,

GRÁFICO 8

México: tipo de cambio real y costo relativo de la mano de obra, 1980-2007

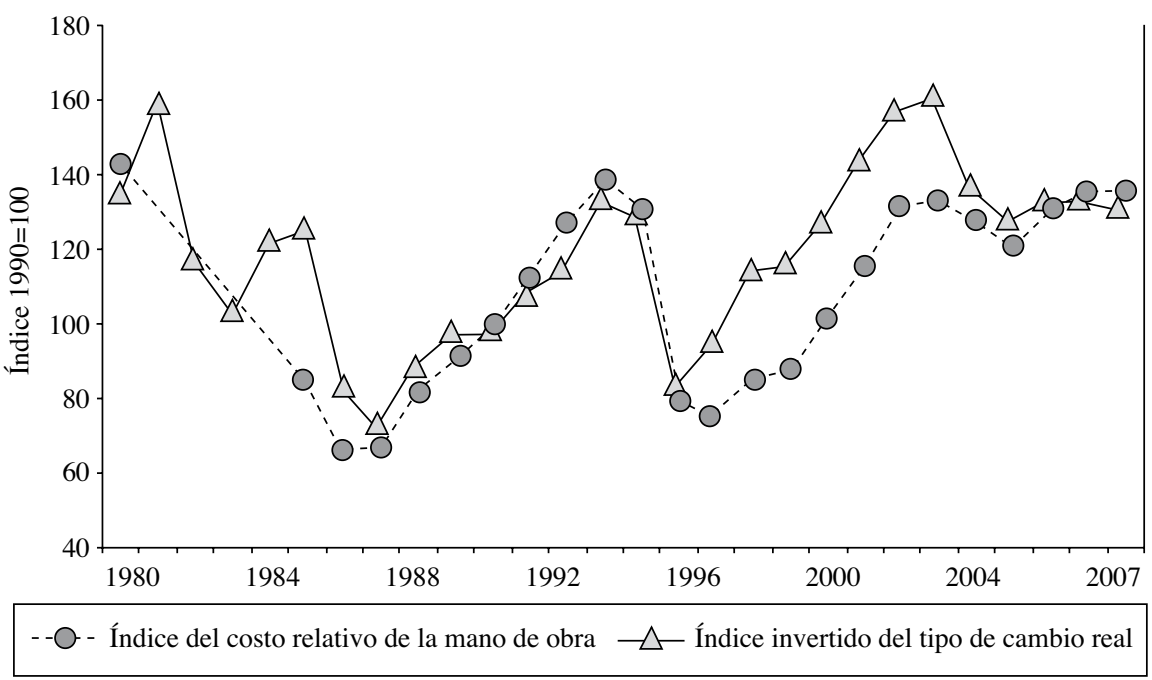

Fuente: cálculos del autor con datos del INEGI (costo laboral relativo) y del Banco de México (tipo de cambio real).

11 Véase en el apéndice la fuente y la definición precisa de las variables incluidas en las regresiones de esta sección. 
México: pruebas de raíz unitaria ${ }^{a}$

\begin{tabular}{|c|c|c|c|c|c|c|}
\hline & \multicolumn{3}{|c|}{ Prueba de Dickey-Fuller aumentada } & \multicolumn{3}{|c|}{ Prueba de Phillips-Perron } \\
\hline & Nivel & $\begin{array}{l}\text { Nivel con } \\
\text { tendencia }\end{array}$ & $\begin{array}{c}\text { Primera } \\
\text { diferencia }\end{array}$ & Nivel & $\begin{array}{l}\text { Nivel con } \\
\text { tendencia }\end{array}$ & $\begin{array}{c}\text { Primera } \\
\text { diferencia }\end{array}$ \\
\hline $\begin{array}{l}\text { Tasa de crecimiento de la exportación de } \\
\text { manufacturas, } \mathrm{GEXP}^{1 /}\end{array}$ & $-2,6625^{*}$ & $-2,8631$ & $-7,0365 * * *$ & $-2,6625^{*}$ & $-2,9800$ & $-8,6171^{* * *}$ \\
\hline Log. del tipo de cambio real, LNRER ${ }^{2 /}$ & $-2,5702^{\mathrm{b}}$ & $-2,4334$ & $-8,1501 * * *$ & $-2,6780 *$ & $-2,7913$ & $-8,2061 * * *$ \\
\hline $\begin{array}{l}\text { Tasa de crecimiento de la producción } \\
\text { industrial de los Estados Unidos, Gus }{ }^{1 /}\end{array}$ & $-2,5470^{\mathrm{b}}$ & $-2,5107$ & $-4,1686^{* * *}$ & $-2,7817^{*}$ & $-2,7473$ & $-5,2266^{* * *}$ \\
\hline $\begin{array}{l}\text { Participación de las ganancias en el valor } \\
\text { agregado de las manufacturas, } \text { PROFIT }^{2 /}\end{array}$ & $-1,1703$ & $-3,4572 * \mathrm{c} 5 /$ & $-9,6741^{* * *}$ & $-1,4044$ & $-2,3108$ & $-9,6889 * * *$ \\
\hline $\begin{array}{l}\text { Tasa de crecimiento de la producción de } \\
\text { manufacturas, } \mathrm{GMPI}^{2 /}\end{array}$ & $-2,0860^{4 /}$ & $-2,2938^{4 /}$ & $-9,5087^{* * * 4 /}$ & $-4,0503 * * *$ & $-3,9804 * *$ & $-10,0838 * * *$ \\
\hline Tasa de crecimiento de la inversión, GINV ${ }^{3 /}$ & $-2,5554^{\mathrm{b} 5 /}$ & $-2,5416^{5 /}$ & $-8,2855^{* * * 5 /}$ & $-3,2852 * *$ & $-3,2640^{*}$ & $-6,7001 * * *$ \\
\hline Tasa de crecimiento del PIB, GGDP ${ }^{3 /}$ & $-3,6762 * * * 6 /$ & $-3,6506 * * 6 /$ & $-8,4493^{* * * 5 /}$ & $-3,3764 * *$ & $-3,3522^{*}$ & $-7,9005 * * *$ \\
\hline Tasa de interés nominal, $\mathrm{NIR}^{3 /}$ & $-4,9442 * * *$ & $-5,3446^{* * *}$ & $-8,9183^{* * *}$ & $-5,1204 * * *$ & $-5,4016^{* * *}$ & $-9,4123 * * *$ \\
\hline Tasa de inflación, $\mathrm{INF}^{3 /}$ & $-5,7769 * * *$ & $-5,9448 * * *$ & $-4,4028 * * *$ & $-4,5717 * * *$ & $-4,0284^{* *}$ & $-4,6306 * * *$ \\
\hline Tasa de ahorro privado, SAVING ${ }^{3 /}$ & $-1,6223$ & $-1,6902$ & $-4,1920 * * *$ & $-2,9508 * *$ & $-2,9967$ & $-21,2476 * * *$ \\
\hline
\end{tabular}

Fuente: estimaciones del autor. Véase en el apéndice la definición y fuente de cada variable.

$* * *, * *, *$ : Hipótesis de raíz unitaria rechazada a $1 \%, 5 \%$ y $10 \%$ de significación estadística.

a Prueba de Dickey-Fuller aumentada con intercepto y longitud de rezago determinada por el criterio de información de Schwartz. Prueba de Phillips-Perron con intercepto, núcleo (kernel) de Bartlett y ancho de banda de Newey-West. Valores críticos de MacKinnon.

b El valor p está entre 0,1 y 0,11 .

c El valor p es 0,0513 .

Nota: la muestra para cada una de las pruebas es igual a la usada en las regresiones correspondientes, aunque en algunos casos su tamaño es menor debido a la inclusión de rezagos en las pruebas:

1/ 1988-T1 2007-T2, 78 observaciones.

2/ 1988-T1 2007-T4, 80 observaciones.

3/ 1988-T1 2007-T3, 79 observaciones.

4/ Reducción de la muestra a 1988-T2 2007-T4, 79 observaciones.

5/ Reducción de la muestra a 1988-T2 2007-T3, 78 observaciones.

6/ Reducción de la muestra a 1988-T3 2007-T3, 77 observaciones.

el período posterior a la liberalización en México. En todos los casos, el punto de partida fue un modelo autorregresivo de rezagos distribuidos, típicamente con cuatro rezagos en la variable dependiente y en los regresores, aunque algunos modelos tuvieron que comenzar con cinco rezagos en la variable dependiente para corregir un problema de autocorrelación de los residuos. La estructura inicial de rezagos fue luego simplificada de acuerdo con la significación estadística de cada coeficiente.

Para asegurar que la simplificación que dio lugar al modelo final sea aceptable en términos estadísticos, todas las regresiones incluyen la prueba de Wald para la hipótesis de que las variables eliminadas tienen coeficientes que en conjunto son iguales a cero. Incluyen también la prueba de Jarque-Bera para la distribución normal de los residuos, la prueba de Breusch-Godfrey para la ausencia de una autocorrelación de cuarto orden, la prueba de Engle para la inexistencia de erro- res de heteroscedasticidad condicional autorregresiva (ARCH) y la prueba RESET de especificación general de Ramsey.

La regresión mediante mínimos cuadrados ordinarios (MCO) de la tasa de crecimiento de la exportación de manufacturas (GEXP) contra el logaritmo del índice del tipo de cambio real basado en el IPC (LNRER), la tasa de crecimiento del índice de la producción industrial de los Estados Unidos (GUS) y dos variables ficticias para captar el incremento temporal - por el inicio del TLC de América del Norte- del crecimiento de la exportación de 1994 y 1995 (o, lo que es equivalente, un aumento permanente del nivel de exportación desde 1994) arroja los resultados siguientes: ${ }^{12}$

\footnotetext{
12 Aunque no es estadísticamente significativo, se retuvo el quinto rezago de GEXP para eliminar un problema de autocorrelación en los residuos.
} 
$G E X P=$

$$
\begin{aligned}
& -0,7074+0,2949 \operatorname{GEXP}(-1)-0,3632 \operatorname{GEXP}(-4)+0,0804 \operatorname{GEXP}(-5)-0,1720 \operatorname{LNRER}(-1) \\
& \begin{array}{llll}
(0,00) & (0,00) \quad(0,00) & (0,39) & (0,00)
\end{array} \\
& +0,3435 \text { LNRER(-4) + 1,0805 GUS + 0,3729 GUS(-4) + 0,0849 NAFTA94 + 0,2235 NAFTA95 } \\
& (0,00) \quad(0,00) \quad(0,07) \quad(0,00) \quad(0,00)
\end{aligned}
$$

Los valores $\mathrm{p}$ se indican entre paréntesis debajo de los coeficientes estimados.

Muestra: primer trimestre de 1988 a segundo trimestre del $2007(n=78)$.

$\mathrm{R}^{2}$ ajustado $=0,8568$.

Estadístico de Jarque-Bera (probabilidad): 0,4826 (0,7856).

Estadístico F de Breusch-Godfrey de cuarto orden (probabilidad): 1,7598 (0,1479)

Estadístico F de efecto ARCH de Engle (probabilidad): 2,4103 (0,1248).

Estadístico F de la prueba RESET de Ramsey (probabilidad): 1,9998 $(0,1619)$.

Estadístico F de la prueba de Wald (probabilidad): 0,9103 (0,5143).

Pruebas de raíz unitaria sobre los residuos de largo plazo (con intercepto; véanse las especificaciones en el cuadro 2).

Estadístico t de la prueba de Dickey-Fuller aumentada (probabilidad): -3,6182 (0,0076).

Estadístico t de la prueba de Phillips-Perron ajustada (probabilidad): -4,4999 (0,0005).

donde la solución de largo plazo es:

$$
\text { GEXP }=-0,7161+0,1736 \text { LNRER }+1,4711 \text { GUS }+0,0859 \text { NAFTA94 + 0,2263 NAFTA95 }
$$

GRÁFICO 9

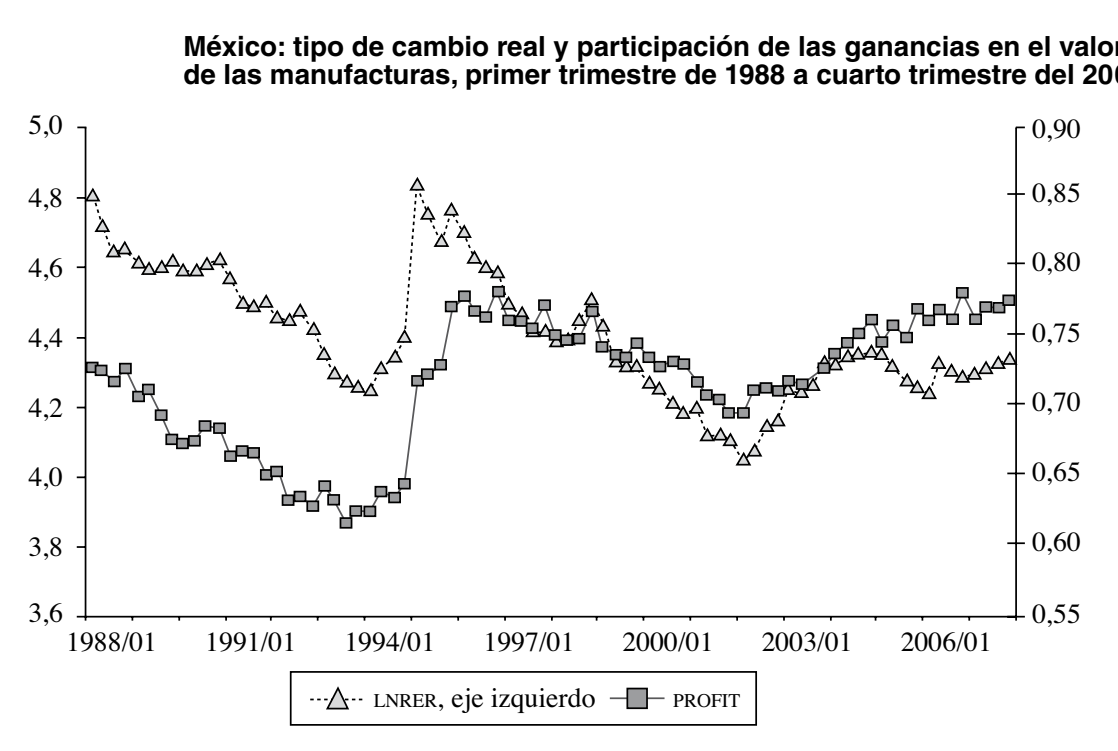

Fuente: cálculos del autor basados en datos del INEGI (Encuesta Industrial Mensual) y del Banco de México (tipo de cambio real).

Los resultados de la estimación confirman la fuerte influencia que tiene la economía estadounidense en la evolución de las exportaciones manufactureras de México. Las variaciones de la tasa de crecimiento del índice de producción industrial de los Estados Unidos inducen variaciones más que proporcionales de la tasa de crecimiento de la exportación. El tipo de cambio real también incide de manera significativa. Por ejemplo, el logaritmo del índice del tipo de cambio real pasó de 4,6329 en 1996 a 4,1108 en el 2002, mientras que el índice de crecimiento de las exportaciones pasó en el mismo lapso de 0,179 a 0,019, es decir, sufrió una merma de 16 puntos porcentuales. El coeficiente estimado del tipo de cambio real permite atribuir 9 puntos de la disminución a la apreciación de la moneda.

Las ecuaciones (5) y (6) implican que una apreciación de la moneda puede tener un efecto negativo en el margen de ganancia y, por ende, en la participación de las ganancias en el PIB. El gráfico 9 revela la estrecha asociación existente entre dicha participación en el caso de 
las manufacturas mexicanas - calculadas como la unidad menos la participación de sueldos y salarios en el valor agregado- y el tipo de cambio real. A comienzos de la década de 1990 y después de la crisis financiera de 1995 , la participación de las ganancias siguió una tendencia descendente al tiempo que la moneda se apreciaba.

Es posible, sin embargo, que los cambios en la participación de las ganancias reflejen el ciclo econó- mico más que las variaciones del tipo de cambio real. Para examinar esta posibilidad, se calculó una regresión, mediante mínimos cuadrados ordinarios, de la participación de las ganancias en las manufacturas (PROFIT) contra la tasa de crecimiento del índice de la producción manufacturera de México (GMPI) y el logaritmo del índice del tipo de cambio real, con los resultados siguientes: ${ }^{13}$

$\begin{array}{cccc}-0,1187+0,8211 & \text { PROFIT }(-1)+0,2863 \text { PROFIT }(-4) & -0,2278 \text { PROFIT }(-5)+0,1430 \text { LNRER } \\ (0,04) & (0,00) & (0,01) & (0,00) \\ -0,0992 & \text { LNRER }(-1)+0,0893 & \text { GMPI }- & 0,1213 \text { GMPI }(-1) \\ (0,00) & (0,01) & (0,00)\end{array}$

Los valores p se indican entre paréntesis debajo de los coeficientes estimados.

Muestra: Segundo trimestre de 1988 a cuarto trimestre del $2007(n=79)$.

$\mathrm{R}^{2}$ ajustado $=0,9611$.

Estadístico de Jarque-Bera (probabilidad): 1,4315 (0,4888).

Estadístico F de Breusch-Godfrey de cuarto orden (probabilidad): 0,3717 (0,8280).

Estadístico F de efecto ARCH de Engle (probabilidad): 0,0679 (0,7952).

Estadístico F de la prueba RESET de Ramsey (probabilidad): 2,0103 (0,1609).

Estadístico F de la prueba de Wald (probabilidad): 1,0365 (0,4195).

Pruebas de raíces unitarias sobre los residuos de largo plazo (con intercepto; véanse las especificaciones en el cuadro 2):

Estadístico t de la prueba de Dickey-Fuller aumentada (probabilidad): -3,3941 $(0,0142)$.

Estadístico t de la prueba de Phillips-Perron ajustada (probabilidad): -4,2871 $(0,0009)$.

donde la solución de largo plazo es:

$$
\text { PROFIT }=-0,9858+0,3637 \text { LNRER }-0,2652 \text { GMPI }
$$

La ecuación (8) muestra que una disminución de la participación de las ganancias en el valor agregado de las manufacturas está asociada a una apreciación de la moneda. ${ }^{14} \mathrm{El}$ efecto estimado es sustancial. Por ejemplo, de acuerdo con la versión de largo plazo de la ecuación, la apreciación de la moneda de 1988-1993 tendió a producir una baja de 15 puntos porcentuales de la participación de las ganancias, en tanto que la disminución real fue de 9,5 puntos porcentuales.

Para tener una idea cuantitativa del efecto sobre la inversión que tiene la participación de las ganancias, utilizando mínimos cuadrados ordinarios se calculó una regresión lineal de la tasa de crecimiento de la inversión fija (GINV) contra la participación de las ganancias en el valor agregado de las manufacturas, la tasa de crecimiento del PIB (GGDP), la tasa de interés nominal (NIR) y la tasa de inflación (INF), obteniéndose: ${ }^{15}$

\footnotetext{
${ }^{13}$ Aunque no se muestran, se incluye una tendencia lineal, habida cuenta de la posible estacionariedad de la participación de las ganancias en torno a ella (véase el cuadro 2), y también variables ficticias trimestrales.

${ }^{14}$ El signo positivo del coeficiente del tipo de cambio real respalda una interpretación de causalidad que va desde el tipo de cambio real a la participación de las ganancias. Supongamos que la causalidad fuera en el sentido opuesto. Si las empresas aumentaran exógenamente sus precios (y, por ende, el margen y la participación de las ganancias), la moneda se apreciaría. El tipo de cambio real y la participación de las ganancias se correlacionarían negativamente.

${ }^{15}$ El hecho de separar la tasa de interés nominal y la tasa de inflación produjo mejores resultados que el uso de su diferencia como medida de la tasa de interés real. La regresión también incluyó una tendencia lineal (véase nota al pie número 13) y, con el fin de lograr la normalidad de los residuos, una variable ficticia para el tercer trimestre de 1989.
} 


$$
G I N V=
$$

$$
\begin{aligned}
& -0,3143+0,4806 \operatorname{GINV}(-1)+0,1118 \operatorname{GINV}(-2)-0,1975 \operatorname{GINV}(-3)-0,6304 \text { PROFIT }+0,8390 \text { PROFIT(-1) } \\
& \begin{array}{lllll}
(0,00) & (0,00) & (0,08) & (0,00) & (0,00)
\end{array} \\
& \begin{array}{ccccccc}
+0,3483 \text { PROFIT(-4) }+ & 2,5105 & G G D P-1,26 & G G D P-0,0021 & N I R+0,0041 & \text { INF }-0,0048 & \text { INF }(-1)+0,0016 \text { INF(-2) } \\
(0,02) & (0,00) & (0,00) & (0,00) & (0,00) & (0,00) & (0,00)
\end{array}
\end{aligned}
$$

Los valores p se indican entre paréntesis debajo de los coeficientes estimados.

Muestra: primer trimestre de 1988 a tercer trimestre del 2007 ( $n=79)$.

$\mathrm{R}^{2}$ ajustado $=0,9606$.

Estadístico de Jarque-Bera (probabilidad): 0,3587 (0,8358).

Estadístico F de Breusch-Godfrey de cuarto orden (probabilidad): 0,1612 (0,9571).

Estadístico F de efecto ARCH de Engle (probabilidad): 2,0129 (0,1601).

Estadístico F de la prueba RESET de Ramsey (probabilidad): 0,0090 $(0,9247)$.

Estadístico F de la prueba de Wald (probabilidad): 0,4312 (0,9435).

Pruebas de raíz unitaria sobre los residuos de largo plazo (con intercepto; véanse las especificaciones en el cuadro 2):

Estadístico t de la prueba de Dickey-Fuller aumentada (probabilidad): $-6,3882(0,0000)$.

Estadístico t de la prueba de Phillips-Perron ajustada (probabilidad): -5,0706 (0,0001).

donde la solución de largo plazo es:

$$
G I N V=-0,5194+0,9204 \text { PROFIT }+2,0669 G G D P-0,0034 N I R+0,0016 I N F
$$

Los coeficientes estimados muestran los signos previstos, con un efecto negativo implícito de la tasa de interés real en el crecimiento de la inversión y un efecto positivo de la tasa de crecimiento del PIB. La participación de las ganancias tiene un efecto positivo con rezago en el crecimiento de la inversión. La magnitud del efecto es significativa. Por ejemplo, la baja de 6,5 puntos porcentuales en la participación de las ganancias durante el período 1996-2002 tendió a reducir la tasa de crecimiento de la inversión en 6 puntos porcentuales o alrededor de un tercio de la reducción efectivamente observada. ${ }^{16}$

Hasta aquí, la apreciación de la moneda tiende a reducir el crecimiento del PIB, no solo por el frecuentemente señalado efecto en el incremento de las exportaciones,

\footnotetext{
${ }^{16}$ En principio, la relación de causalidad entre rentabilidad e inversión puede funcionar en ambos sentidos. Como hace mucho señaló Kalecki (1942), un aumento de la inversión puede elevar las ganancias por su efecto en la demanda agregada. La ecuación (9a) capta un efecto diferente, con rezago, de las ganancias sobre la inversión.
}

sino también por su efecto en la participación de las ganancias en el PIB y el aumento de la inversión. Existe un posible efecto compensatorio. Si la tasa de ahorro de los trabajadores es menor que la de los inversores, una apreciación de la moneda tenderá a reducir la tasa total de ahorro al incrementar la participación del trabajo en el ingreso.

Un examen somero de los gráficos 5 y 8 sugiere que efectivamente existe una estrecha conexión entre el tipo de cambio real y la tasa de ahorro privado de México. La tasa de ahorro también podría reaccionar ante variaciones de la tasa de crecimiento del PIB. Para separar estos efectos, se calculó por el método de mínimos cuadrados ordinarios una regresión de la tasa de ahorro privado (SAVING) ${ }^{17}$ contra el tipo de cambio real y la tasa de crecimiento del PIB, obteniéndose:

\footnotetext{
${ }^{17}$ Esta tasa es similar a la empleada en la ecuación (2), pero habiendo eliminado los efectos estacionales mediante una regresión contra un conjunto de variables ficticias trimestrales.
} 


$$
\begin{aligned}
& \text { - 0,0518 + 0,5607 SAVING(-1) + 0,6525 SAVING(-4) - 0,3387 SAVING(-5) + 0,0182 LNRER } \\
& \begin{array}{llll}
(0,14) \quad(0,00) & (0,00) & (0,00)
\end{array} \\
& \text { - 0,1628 GGDP(-2) + 0,2077 GGDP(-3) - 0,1708 GGDP(-4) } \\
& (0,02) \quad(0,02) \quad(0,01)
\end{aligned}
$$

Los valores p se indican entre paréntesis debajo de los coeficientes estimados.

Muestra: primer trimestre de 1988 a tercer trimestre del $2007(n=79)$.

$\mathrm{R}^{2}$ ajustado $=0,7924$.

Estadístico de Jarque-Bera (probabilidad): 0,1026 (0,95).

Estadístico F de Breusch-Godfrey de cuarto orden (probabilidad): 0,8229 (0,5152).

Estadístico F de efecto ARCH de Engle (probabilidad): 0,9254 (0,3391).

Estadístico F de la prueba RESET de Ramsey (probabilidad): 2,4162 (0,1246).

Estadístico F de la prueba de Wald (probabilidad): 1,5523 (0,1576).

Pruebas de raíz unitaria sobre los residuos de largo plazo (con intercepción; véanse las especificaciones en el cuadro 2):

Estadístico t de la prueba de Dickey-Fuller aumentada (probabilidad): -3,1473 $(0,0272)$.

Estadístico t de la prueba de Phillips-Perron ajustada (probabilidad): -3,1473 $(0,0272)$.

donde la solución de largo plazo es:

$$
\text { SAVING }=-0,4125+0,145 \text { LNRER }-1,0026 \text { GGDP }
$$

La tasa de ahorro privado parece comportarse en forma anticíclica (con rezago), resultado algo anómalo a la luz de las teorías del consumo basadas en el ingreso permanente, pero que podría explicarse por la existencia de restricciones del crédito al consumo. Como se preveía, el tipo de cambio real tiene un marcado efecto en la tasa de ahorro. Por ejemplo, de acuerdo con el coeficiente del tipo de cambio real de largo plazo, la apreciación de $52 \%$ entre 1996 y el 2002 tendió a reducir la tasa de ahorro en unos 7,5 puntos porcentuales, cifra que está un punto porcentual por encima de la reducción efectivamente observada. ${ }^{18}$

Los resultados muestran que la apreciación de la moneda tiene efectos parciales en la demanda agregada que actúan en direcciones opuestas: por un lado, la deprime por su efecto negativo en la balanza comercial y la rentabilidad de la inversión, y por otro, la eleva por

\footnotetext{
${ }^{18}$ Aquí corresponde un comentario sobre la causalidad similar al que se hizo respecto de la ecuación de PROFIT (véase nota al pie número 14). Supongamos que la tasa de ahorro aumentara en forma exógena. El saldo de la cuenta corriente también aumentaría y, si existiera un efecto sustancial que actuara por este conducto, la moneda se apreciaría. En este caso, el tipo de cambio real y la tasa de ahorro estarían correlacionados negativamente.
}

una caída de la tasa de ahorro. En principio, el resultado neto es incierto. ${ }^{19}$

En el caso concreto de México, sobre todo desde que rige el TLC de América del Norte, la reducción de la tasa de ahorro por la apreciación de la moneda ha sido compensada con creces por el aumento de la participación de las importaciones en el PIB (presumiblemente, debido a la apreciación misma y a la liberalización del régimen de comercio). Como resultado, bajó el multiplicador de la demanda. Esto, unido al lento avance de la inversión, produjo una trayectoria plana de los niveles de inversión ajustados por el multiplicador y un aporte casi nulo de esta variable al crecimiento del PIB.

Nuestro análisis se ha centrado en la participación de las ganancias en el PIB. Sin embargo, el comportamiento de la tasa de ganancia (ganancias/capital) $r$ depende no solo de la participación de las ganancias (ganancias/PIB), sino también de la razón PIB/capital $k$, según la definición:

$$
r=z k
$$

\footnotetext{
${ }^{19}$ Véase un análisis teórico del tema en Blecker (2002).
} 
Es difícil medir la razón PIB/capital debido a la falta de series prolongadas de acervos de capital. Sin embargo, es posible estimarla a partir de la ecuación siguiente:

$$
g=i k_{m}
$$

donde $g$ es la tasa de crecimiento del PIB, $i$ es la tasa de inversión (inversión fija/PIB) y $k_{m}$ es la razón producto/ capital. $^{20}$

Las observaciones de $k_{m}$ fueron derivadas como residuos de las series anuales de la tasa de crecimiento del PIB y la tasa de inversión. A esta frecuencia, las series pueden ser muy variables por las fluctuaciones de la demanda agregada y, por tanto, del grado de utilización de la capacidad. Las fluctuaciones fueron eliminadas tomando las tendencias Hodrick-Prescott de las series o bien sus promedios en períodos prolongados.

El gráfico 10 presenta las tendencias Hodrick-Prescott correspondientes a 1960-2007 y muestra que hubo una disminución de la razón PIB/capital en los decenios de 1960 y 1970 y a comienzos de los años 1980. Como era de prever, el mismo patrón acusan los promedios por períodos que aparecen en el cuadro 3. La razón media PIB/capital descendió de 0,35 en 1960-1977 a 0,22 a comienzos de la década de 1990 y a 0,18 a partir de $1996 .{ }^{21}$
Como estamos centrándonos en las tendencias Hodrick-Prescott y en las variaciones medias de largo plazo, el descenso de la razón PIB/capital debería tener una base tecnológica. Puede deberse también a un problema persistente de subutilización del capital instalado, como lo sugieren los resultados de las encuestas de opinión realizadas por el Banco de México entre gerentes del sector manufacturero (López, s/f). En este caso, la demanda agregada habría dejado reiteradamente de crecer a las tasas previstas por las empresas al adoptar sus decisiones de inversión.

\section{CUADRO 3 \\ México: identidad del crecimiento \\ (Promedios anuales)}

\begin{tabular}{cccc}
\hline & $\begin{array}{c}\text { Tasa de crecimiento } \\
\text { del PIB }\end{array}$ & $\begin{array}{c}\text { Tasa de } \\
\text { inversión }\end{array}$ & $\begin{array}{c}\text { Razón } \\
\text { PIB/capital }^{\mathrm{a}}\end{array}$ \\
\hline $1960-1977$ & 6,23 & 18,14 & 0,35 \\
$1989-1994$ & 3,91 & 18,04 & 0,22 \\
$1996-2007$ & 3,58 & 19,80 & 0,18 \\
\hline
\end{tabular}

Fuente: datos de cuentas nacionales del INEGI y Banco Mundial (2005).

a Véase la ecuación (12) en el texto para el cálculo de la razón $\mathrm{PIB} /$ capital.

GRÁFICO 10

México: identidad del crecimiento, 1960-2007, tendencias de Hodrick-Prescott

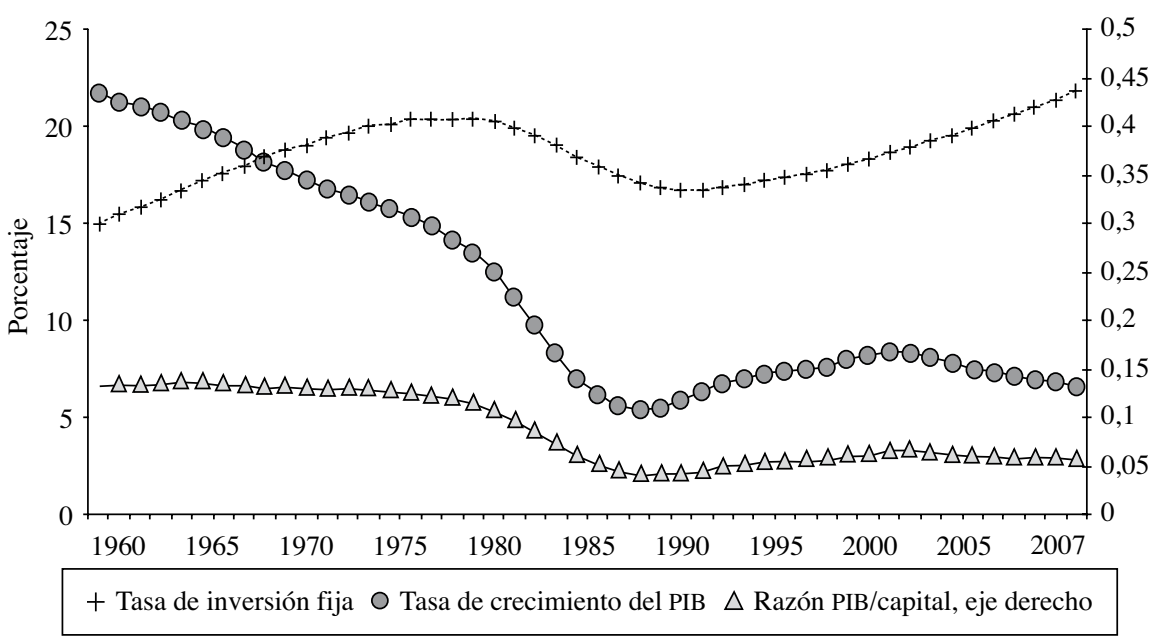

Fuente: estimaciones del autor con datos de cuentas nacionales del Banco Mundial (2005) y del INEGI.

\footnotetext{
${ }^{20}$ Una limitación estriba en que la tasa de ganancia depende de la razón PIB/capital promedio, en tanto que la ecuación (12) produce una estimación de la razón marginal.

21 Trabajando dentro de un contexto analítico diferente, Santaella (1998) y De Gregorio y Lee (1999) presentan pruebas de que el creci-
}

miento de la productividad total de los factores en México disminuyó notablemente a partir de los años 1970, llegando a tasas negativas en los decenios de 1980 y 1990 . Esto implica un efecto negativo sobre la tasa de ganancia. 
Pero ya se trate de un fenómeno tecnológico o el resultado de insuficiencia en la demanda agregada, al disminuir la razón Рів/capital no disminuye solo la tasa de crecimiento del PIB (ecuación 12),

\section{V}

\section{Conclusiones}

A fines de la década de 1980 México se transformó en lugar de destino de ingentes corrientes de capital y en un gran exportador de manufacturas, pero no logró sostener un crecimiento económico elevado. Los analistas de la economía mexicana han ofrecido diversas explicaciones de esta aparente paradoja. En este trabajo se argumenta que es útil considerar una historia simple de precios relativos y rentabilidad de la inversión.

Desde fines de los años 1980, la gestión macroeconómica de México y particularmente su política monetaria se centraron en reducir la inflación. La parte medular del proceso duró más de 15 años, debido entre otras causas al revés temporal que significó la crisis financiera de 1995.

A medida que avanzaba el proceso de desinflación, el peso se apreciaba en términos reales. En general, la apreciación de la moneda reduce directamente la tasa de crecimiento del PIB por su efecto en la exportación neta, como lo atestigua la desaceleración de las exportaciones a comienzos de los años 2000. Pero la apreciación puede también reducir indirectamente el crecimiento del PIB, por su efecto en la participación de las ganancias en el PIB. En este artículo se considera la vía indirecta en el caso de México, y se presenta evidencia econométrica sino también la tasa de ganancia (ecuación 11). Esto agrava el efecto que la apreciación del peso ejerce sobre la rentabilidad a través de la participación de las ganancias en el PIB. de los efectos significativos del tipo de cambio real sobre la participación de las ganancias en el sector manufacturero, y de la participación de las ganancias sobre la inversión.

La tasa de ganancia depende tanto de la participación de las ganancias en el PIB como de la razón PIB/ capital. En el trabajo se mostró que la razón (marginal) $\mathrm{PIB} /$ capital en los años de crecimiento lento ha estado bastante por debajo de los niveles observados en México en su período de crecimiento rápido, antes de la década de 1980. Por definición, la merma de la razón PIB/capital reduce la tasa de crecimiento del PIB cualquiera sea la tasa dada de inversión, pero también tiende a reducir la tasa de inversión misma, por sus efectos en la tasa de ganancia.

No es de extrañar entonces que la mayor parte del crecimiento del PIB provenga de la expansión de las exportaciones, como lo demuestra una simple descomposición del PIB por el lado de la demanda, mientras que el aporte de la inversión ha sido insignificante. El crecimiento lento de la economía mexicana es en parte una historia de inversión poco dinámica y baja rentabilidad en una situación de desinflación y apreciación real de la moneda.

APÉNDICE

Definiciones y fuente de los datos

Gráfico 2: se calcularon las tasas de importación específicas como importación de bienes intermedios/PIB, importación de bienes de consumo/consumo agregado e importación de bienes de capital/inversión fija. Fuente: datos originales de comercio del Banco de México y datos de las cuentas nacionales del Instituto Nacional de Estadística, Geografía e Informática (INEGI).

GEXP: variación en cuatro trimestres de la exportación real de manufacturas. Los datos originales de balanza de pagos en dólares corrientes fueron deflactados por el índice de precios al productor de los Estados Unidos. Fuente: Banco de México y Oficina de estadísticas del trabajo de los Estados Unidos.

LNRER: logaritmo natural del índice del tipo de cambio efectivo real basado en el IPC. Fuente: índice original mensual del Banco de México.

GUS: variación en cuatro trimestres del índice de producción industrial de Estados Unidos, desestacionalizado. Fuente: índice original mensual de la Reserva Federal de los Estados Unidos. 
PROFIT: promedio trimestral de la participación de las ganancias, calculada como la unidad menos la razón sueldos y salarios/valor agregado en el sector de las manufacturas. En el cálculo se usó la razón media entre el valor agregado y la producción bruta, según datos de la Encuesta Industrial Anual del INEGI correspondiente al período más prolongado disponible (1994-2003), para calcular el valor agregado a partir del índice mensual. Fuente: datos originales mensuales en pesos corrientes extraídos de la Encuesta Industrial Mensual del INEGI.

GMPI: variación en cuatro trimestres del índice de producción manufacturera. Fuente: índice original mensual del INEGI.

GINV: variación en cuatro trimestres de la inversión fija bruta real. Fuente: datos originales en miles de pesos de 1993, INEGI.

GGDP: variación en cuatro trimestres del producto interno bruto real. Fuente: datos originales en miles de pesos de 1993, INEGI.
NIR: promedio trimestral de la tasa a 91 días, anualizada, de los Certificados de la Tesorería de México (CETES), como porcentaje. Fuente: datos originales mensuales del Banco de México.

INF: variación en cuatro trimestres del índice medio de precios al consumidor, como porcentaje. Fuente: índice original mensual de precios al consumidor, Banco de México.

SAVING: la unidad menos la razón entre el consumo privado, descontados los impuestos, y el PIB. El cálculo incluye todo el ingreso del sector público de actividades económicas no petroleras clasificado como impuesto, más los impuestos a la gasolina. La variable empleada en la ecuación (10a) es el residuo de una regresión de la tasa de ahorro registrada sobre un conjunto de variables ficticias trimestrales (ajustada por el intercepto estimado). Fuente: datos originales en miles de pesos de 1993, INEGI.

\section{Bibliografía}

Banco Mundial (2005): World Development Indicators, 2005, Washington, D.C., versión en CD-Rom.

Berg, J. y L. Taylor (2000): External Liberalization, Economic Performance and Social Policy, CEPA working paper, $\mathrm{N}^{\circ} 12$, Nueva York, New School University, febrero.

Bergoeing, R. y otros (2001): A decade lost and found: Mexico and Chile in the 1980s, documento preparado para una conferencia organizada por el Banco de la Reserva Federal de Minneapolis, septiembre.

Blecker, R. (2002): Distribution, demand and growth in neo-Kaleckian macro-models, The Economics of Demand-led Growth, Mark Setterfield, Cheltenham, Reino Unido, Edward Elgar. (2007): External Shocks, Structural Change, and Economic Growth in Mexico, 1979-2006, documento de trabajo, Washington, D.C., Departamento de Economía, American University, diciembre.

Blyde, J. S. y E. Fernández-Arias (2004): Why does Latin America grow more slowly?, Economic and Social Studies, Washington, D.C., Banco Interamericano de Desarrollo.

De Gregorio, J. y J-W. Lee (1999): Economic growth in Latin America: sources and prospects, documento preparado para la Global Development Network, diciembre.

Feenstra, R. y H. L. Kee (2007): Trade liberalization and export variety: a comparison of Mexico and China, The World Economy, vol. 30, $\mathrm{N}^{\mathrm{o}}$ 1, Oxford, Blackwell Publishing.

Galindo, L. M. y J. Ros (2008): Alternatives to inflation targeting in Mexico, International Review of Applied Economics, vol. 22, $\mathrm{N}^{\circ}$ 2, Londres, Routledge.

Hausmann, R., J. Hwang y D. Rodrik (2007): What you export matters, Journal of Economic Growth, vol. 12, No 1, Nueva York, Springer.

Ibarra, C. (2008a): Disinflation and real currency appreciation in Chile and Mexico: the role of monetary policy, próxima publicación en Investigación Económica, Facultad de Economía, Universidad Nacional Autónoma de México.

(2008b): Exporting without growing: investment, real currency appreciation, and export-led growth in Mexico, documento de trabajo, Puebla, Departamento de Economía, Universidad de las Américas Puebla, junio.
(2003): Sluggish growth, trade liberalization and the Mexican disease: a medium-term macroeconomic model with an application to Mexico, International Review of Applied Economics, vol. 17, $\mathrm{N}^{\mathrm{o}}$ 3, Londres, Routledge.

Kalecki, M. (1942): A theory of profits, The Economic Journal, vol. $52, \mathrm{~N}^{\circ} 206 / 207$.

Kamin, S. y J. Rogers (2000): Output and the real exchange rate in developing countries: an application to Mexico, Journal of Development Economics, vol. 61, № 1 , Amsterdam, Elsevier.

Lall, S. (2000): The Technological Structure and Performance of Developing Countries Manufactured Exports, QEH working paper, $\mathrm{N}^{\mathrm{o}} 44$, Oxford, Universidad de Oxford, junio.

Lederman, D., A. M. Menéndez y otros (2003): Mexican investment after the Tequila crisis: basic economics, 'confidence' effects or market imperfections?, Journal of International Money and Finance, vol. 22, $\mathrm{N}^{\mathrm{o}}$ 1, Amsterdam, Elsevier.

López, J. (s/f): Mexico's economic prospects reconsidered, documento de trabajo, México, D.F., Universidad Nacional Autónoma de México.

López, J. y A. Cruz (2000): 'Thirlwall's law' and beyond: the Latin American experience, Journal of Post Keynesian Economics, vol. 22, $\mathrm{N}^{\mathrm{o}} 3$, Nueva York, M.E. Sharpe Inc.

Moreno-Brid, J. C. (1999): Mexico's economic growth and the balance of payments constraint: a cointegration analysis, International Review of Applied Economics, vol. 13, $\mathrm{N}^{\circ} 2$, Londres, Routledge.

Moreno-Brid, J. C., J. Santamaría y J. C. Rivas (2005a): Industrialization and economic growth in Mexico alter NAFTA: the road travelled, Development and Change, vol. 36, $\mathrm{N}^{\circ} 6$, Oxford, Blackwell Publishing. (2005b): Mexico: Economic Growth, Exports and Industrial Performance after NAFTA, serie Estudios y perspectivas, $\mathrm{N}^{\circ} 42$, LC/L.2479-P, México, D.F., Santiago de Chile, Sede Subregional de la CEPAL en México. Publicación de las Naciones Unidas, $\mathrm{N}^{\mathrm{o}}$ de venta: E.06.II.G.6.

Pacheco-López, P. (2005): The impact of trade liberalization on exports, imports, the balance of payments and growth: the case of Mexico, Journal of Post Keynesian Economics, vol. 27, № 4, Nueva York, M.E. Sharpe Inc. 
Ramos, M. y A. Torres (2005): Reducing inflation through inflation targeting. The Mexican experience, documento de trabajo, México, D.F., Banco de México, julio.

Ros, J. y N. Lustig (2000): Trade and Financial Liberalization with Volatile Capital Inflows: Macroeconomic Consequences and Social Impacts in Mexico during the 1990s, CEPA working paper, $N^{\circ} 18$, Nueva York, New School University, febrero.
Ros, J., J. Draisma y otros (1996): Prospects for growth and the environment in Mexico in the 1990s, World Development, vol. 24, $\mathrm{N}^{\circ}$ 2, Amsterdam, Elsevier.

Santaella, J. (1998): Economic growth in Mexico. Searching for clues to its slowdown, documento preparado para el Banco Interamericano de Desarrollo, diciembre.

Tornell, A., F. Westermann y L. Martínez (2004), NAFTA and Mexico's Less than Stellar Performance, NBER working paper, $\mathrm{N}^{\circ} 10289$, Cambridge, Massachusetts, National Bureau of Economic Research, febrero. 